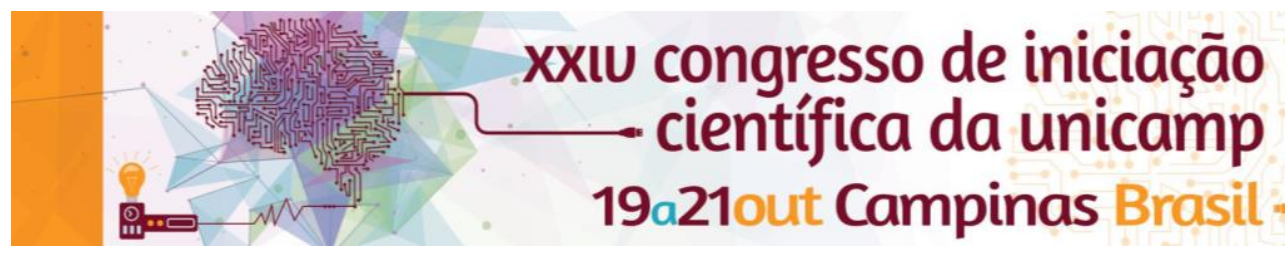

\title{
Computer vison for classification and quality analysis of food.
}

\author{
Matheus G. A. Sasso*, Marcio Schmiele, Maria Tersa Silva Clerici, Sylvio Barbon Jr., Douglas Fernandes \\ Barbin
}

Abstract

It was investigated the classification of diferent kinds of pasta according to their composition by image analyses

\author{
Key words: \\ Computer vision, Image Analysis, classification.
}

\section{Introduction}

Overview:

Nowadays, the techniques to evaluate quality and safety of food are blooming. One of them is the use of computer vision, which is efficient because is a non destructive method, where samples can be analysed in a non-contact manner, making it still available for futher application.

Computer vision techniques are based on sample aspects, such as color, texture and others.

Objectives:

- Prepare the equipment for the minimum interferance of light in the image aquisition.

- Acquisition of imagens with a microscope.

- Segmentation of the images.

- Extracting image descriptors as color ${ }^{1}$ and texture ${ }^{2}$ using image analysis and exports the information to an Excel sheet, using MATLAB codes.

- Apply statistics methods to classify the diferent kinds of pasta.

\section{Results and Discussion}

Description and discussion of the results:

The results from the statistics analyses show us that the difference in some aspects are related to the different kinds of components in pasta.

Using the image parameters obtained, classification of samples was performed.

Important details about methodology:

In order to apply the image segmentation step, the Otsu filter was used.

Some descriptors were applied on the full image, others in cropped image and some in grayscale images, according with the algorithm efficiency.

One way ANOVA was used to analyse the difference between samples according to the image parameters.

The best parameter for classification of samples was the RGB color extracted from samples, as they are related to some major components in the samples.

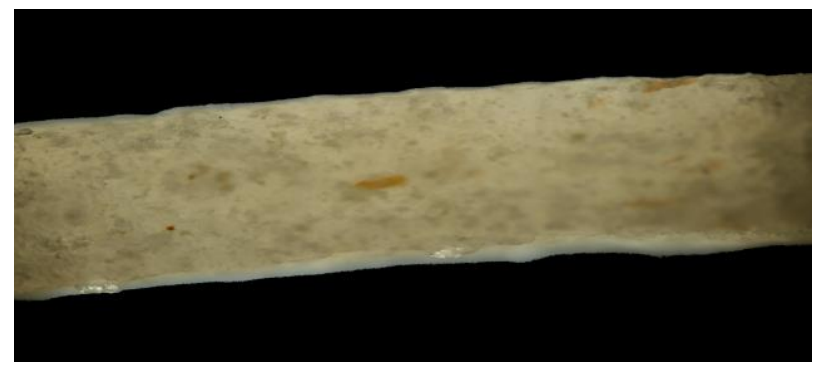

Image 1. Example of segmented image of pasta

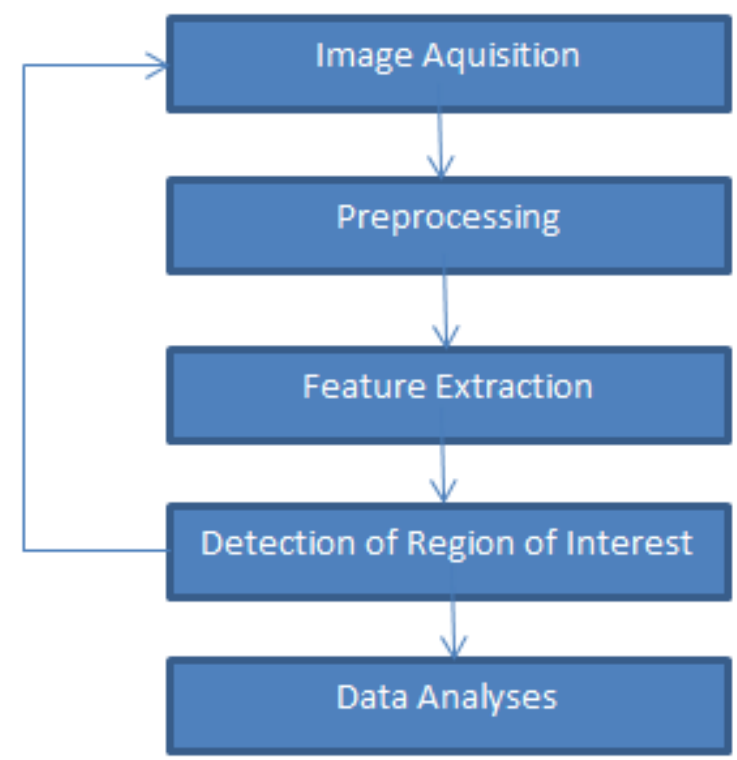

Image2. Image processing flowchart

\section{Conclusions}

The proposed approach has provided useful results, showing that computer vision could be further investigated for applications in the food processing industry

\section{Acknowledgement}

The authors would like to acknowledge CNPq (National Counsel of Technological and Scientific Development) represented by PIBIC/PRP Unicamp for the financial support; and UEL (State University of Londrina) for scientific contribution and learning experience.

[1] Colour calibration of a laboratory computer vision system for quality evaluation of pre-sliced hams Nektarios A. Valous, Fernando Mendoza, DaWen Sun, Paul Allen

[2] Surface texture characterization of an Italian pasta by means of univariate and multivariate feature extraction from their texture images Lorenzo Fongaro, Knut Kvaal 